\title{
LncRNA-ECM is overexpressed in esophageal squamous cell carcinoma and promotes tumor metastasis
}

\author{
JUAN YAO $^{1 *}$, XIAOZHOU SHEN ${ }^{1 *}$, HONGZHI LI ${ }^{1}$, JIE XU ${ }^{1}$, \\ SHANSHAN SHAO ${ }^{1}$, JUN-XING HUANG ${ }^{1}$ and MEI LIN ${ }^{2}$ \\ ${ }^{1}$ Department of Oncology, Taizhou People's Hospital Affiliated to Nantong University; \\ ${ }^{2}$ Institute of Clinical Medicine, Taizhou People's Hospital Affiliated to Nantong University, Taizhou, Jiangsu 225300, P.R. China
}

Received November 30, 2017; Accepted June 13, 2018

DOI: $10.3892 / 01.2018 .9130$

\begin{abstract}
The aim of the present study was to investigate the expression of long non-coding(lnc) RNA-extracellular matrix $(E C M)$ in esophageal squamous cell carcinoma (ESCC) and its effect on ESCC metastasis. Using reverse transcription-quantitative polymerase chain reaction (RT-qPCR), the expression of IncRNA-ECM in ESCC tissues was investigated and compared with that in corresponding adjacent tissues. In addition, the expression of IncRNA-ECM in the human ESCC cell lines TE-1, EC9706, KYSE150, Eca109 and KYSE30 was also detected and compared with that in the normal esophageal mucosal epithelial cell line HET-1A. The clinicopathological association between IncRNA-ECM and ESCC was assessed. Silencing and overexpression of lncRNA-ECM in ESCC TE-1 and Eca109 cells determined the correlation between IncRNA-ECM expression and ESCC invasion and metastasis. The possible target genes of lncRNA-ECM were predicted and verified by bioinformatics analysis and experimental results. The expression level of intercellular adhesion molecule 1 (ICAM1) was detected in ESCC tissues by RT-qPCR and the correlation between the expression of ICAM1 and lncRNA-ECM was analyzed. Changes in the expression of ICAM1 in ESCC TE-1 and Eca109 cell lines were evaluated after knocking down lncRNA-ECM and transfection of lncRNA-ECM overexpression plasmids. The expression level of IncRNA-ECM in the tissues of ESCC
\end{abstract}

Correspondence to: Dr Jun-Xing Huang, Department of Oncology, Taizhou People's Hospital Affiliated to Nantong University, 210 Yingchun Road, Taizhou, Jiangsu 225300, P.R. China

E-mail:hjxtz@sina.cn

Dr Mei Lin, Institute of Clinical Medicine, Taizhou People's Hospital Affiliated to Nantong University, 210 Yingchun Road, Taizhou, Jiangsu 225300, P.R. China

E-mail:1_mei@163.com

*Contributed equally

Key words: long non-coding RNA ENST00000589379, esophageal squamous cell carcinoma, metastasis with lymph node metastasis were significantly increased compared with ESCC with no lymph metastasis $(\mathrm{P}<0.05)$. LncRNA-ECM silencing notably reduced the invasion and metastasis of TE-1 and Eca109 cells, while lncRNA-ECM overexpression promoted the invasion and metastasis of the two cell lines. The expression level of ICAMI was directly correlated with the expression of IncRNA-ECM, suggesting that ICAM1 may be the downstream target gene of IncRNA-ECM. LncRNA-ECM was revealed as being overexpressed in ESCC. LncRNA-ECM expression was positively correlated with metastasis and may affect the metastasis of ESCC through ICAMI regulation. These findings indicate that IncRNA-ECM may be promising as a novel biomarker for the diagnosis and prediction of prognosis for ESCC, and it may also serve as a novel therapeutic target for ESCC.

\section{Introduction}

Esophageal cancer is one of the most common malignancies, with an incidence rate that ranks sixth in China and fourth among the causes of cancer-related mortality (1). Esophageal cancer is also one of the most common lethal tumors worldwide with the sixth highest mortality rate (2). Esophageal squamous cell carcinoma (ESCC) accounts for $>90 \%$ of the cases of esophageal cancer, and has a high degree of aggressiveness and complexity of biological behavior, making its clinical course variable and heterogeneous. A considerable number of ESCC patients have already metastasized at diagnosis. The metastasis of ESCC is the main cause of poor prognosis and mainly involves lymph node and distant metastasis (3). Previous studies demonstrated that the 5-year survival rate of ESCC patients without metastasis was $70-92 \%$, whereas it was only $18-47 \%$ in those with metastasis $(3,4)$. Therefore, an in-depth understanding of the mechanism underlying ESCC metastasis is of high scientific and clinical value for improving the survival rate of ESCC.

Long non-coding $(\operatorname{lnc})$ RNAs are a class of mature RNAs with a transcription length of $>200$ bases, which is significantly longer compared with that of mRNA. Despite having no coding ability, IncRNAs are involved in several processes, including gene imprinting, chromatin remodeling, cell cycle regulation, splicing regulation, mRNA degradation and translation regulation (5). They can regulate gene expression at the genetic, transcriptional and post-transcriptional level, 
and are widely implicated in most physiological as well as pathological processes (6). An increasing number of studies have demonstrated that differentially expressed lncRNAs are closely associated with tumor proliferation, invasion, metastasis and prognosis, and have great potential of becoming new targets for tumor therapy $(7,8)$. It was demonstrated that IncRNAs are closely related to ESCC (9-11). However, little is known on their effect on ESCC metastasis. Therefore, it is crucial to identify differentially expressed lncRNAs in ESCC and investigate their function and underlying mechanism in ESCC metastasis.

LncRNA-ENST00000589379 has been found to be highly expressed in ESCC tissues with undefined function by gene chips (12). In the present study, this novel lncRNA is referred to as IncRNA-ECM. To further investigate the role of lncRNA-ECM in ESCC metastasis, we investigated the correlation between its expression and the clinical pathology of ESCC using real-time fluorescence quantitative PCR. Changes in cell invasion and migration capacity were detected following lncRNA-ECM knockdown/overexpression in the ESCC TE-1 and Eca109 cell lines by classical Transwell assay. Bioinformatics analysis and rescue experiments were used to examine the expression changes in the downstream geneintercellular adhesion molecule 1 (ICAM1) in order to elucidate the mechanism of action of IncRNA-ECM.

\section{Materials and methods}

Cases and specimens. Tissue samples from 62 cases with ESCC and matched paracancerous tissues were collected in Taizhou People's Hospital affiliated to Nantong University (Nantong, China) between September 2014 and December 2016. All the cases were confirmed by pathological examination. The patients included 43 men and 19 women, with a mean age at surgery of $65.75 \pm 6.39$ years. None of the patients had received any radiotherapy or chemotherapy prior to surgery. The fresh specimens were immediately placed in liquid nitrogen; 8-10 min later, they were packed into RNAfixer (Takara, Dalian, China) and preserved at $-80^{\circ} \mathrm{C}$. The tumors were classified according to the 7 th edition of the American Joint Committee on Cancer TNM staging guidelines for esophageal cancer. The study was approved by the Ethics Committee of Taizhou People's Hospital affiliated to Nantong University and the informed consent of the patients was obtained.

$R N A$ extraction and RT- $q P C R$. Total RNA was extracted from ESCC samples and ESCC cell lines using the TRIzol reagent kit (Invitrogen; Thermo Fisher Scientific, Inc., Waltham, MA, USA) in accordance with the manufacturer's protocol; The total RNA quality and concentration was determined by UV spectrophotometry. Double-stranded complementary DNA was synthesized by reverse transcription in accordance with the cDNA synthesis kit; qPCR was performed using the Script SYBR Green PCR kit (both Toyobo, Osaka, Japan). The primer sequences of IncRNA-ECM were as follows: Forward, 5'-CAA TATGTCCGTGGGACCCT-3' and reverse, 5'-GGAGGCCAA ACAACTGTGGA-3'. The primer sequences for ICAM1 were as follows: Forward, 5'-TTGAGGGCACCTACCTCTGT-3' and reverse, 5'-GATAGGTTCAGGGAGGCGTG-3'. $\beta$-actin was used as reference (forward, 5'-CTGGGACGACATGGA
Table I. Association between long non-coding RNA-ECM expression and the clinicopathological features of patients with ESCC.

\begin{tabular}{|c|c|c|c|}
\hline Variable & Patients & Mean \pm SD & P-value \\
\hline Age (years) & & & 0.528 \\
\hline$\geq 60$ & 52 & $4.64 \pm 1.58$ & \\
\hline$<60$ & 10 & $5.26 \pm 1.68$ & \\
\hline Sex & & & 0.744 \\
\hline Male & 43 & $4.91 \pm 1.51$ & \\
\hline Female & 19 & $4.35 \pm 1.76$ & \\
\hline Tumor size $(\mathrm{cm})$ & & & 0.867 \\
\hline$\leq 3$ & 25 & $4.51 \pm 1.78$ & \\
\hline$>3$ & 37 & $4.89 \pm 1.47$ & \\
\hline $\begin{array}{l}\text { Pathological } \\
\text { differentiation grade }\end{array}$ & & & 0.609 \\
\hline Well & 6 & $4.75 \pm 1.53$ & \\
\hline Moderately & 35 & $4.74 \pm 1.55$ & \\
\hline Poorly & 21 & $4.72 \pm 1.77$ & \\
\hline T stage & & & 0.471 \\
\hline $\mathrm{T} 1-2$ & 30 & $4.37 \pm 1.61$ & \\
\hline $\mathrm{T} 3-4$ & 32 & $5.08 \pm 1.54$ & \\
\hline N stage & & & 0.029 \\
\hline No & 38 & $4.20 \pm 1.56$ & \\
\hline N1 & 24 & $5.59 \pm 1.27$ & \\
\hline TNM stage & & & 0.036 \\
\hline I-II & 43 & $4.23 \pm 1.54$ & \\
\hline III-IV & 19 & $5.87 \pm 1.07$ & \\
\hline
\end{tabular}

SD, standard deviation; ECM, extracellular matrix; TNM, tumor, node, metastasis; ESCC, esophageal squamous cell carcinoma.

GAAAA-3' and reverse, 5'-AAGGAAGGCTGGAAGAGT GC-3'). Each experiment was duplicated three times. Relative expression levels were calculated using the $2^{-\Delta \Delta \mathrm{Ct}}$ method.

Microarray analysis (12). LncRNA gene chip service was provided by Outdo Biotech [Agilent Human IncRNA Microarray V2.0 (4x180K; design ID 062918; containing 46,506 lncRNAs); Agilent Technologies, Inc., Santa Clara, CA, USA]. The operating process of the gene chip was as follows: First, RNA was prepared and double-stranded cDNA was synthesized by reverse transcription; next, a fluorescently labeled cRNA was synthesized with cyanine-3-CTP (Cy3). Then, chip hybridization between the cRNA and the chip was conducted after measuring their concentration and purity. After elution, the original image was obtained by scanning through the Agilent Scanner G2505C (Agilent Technologies, Inc.). The Feature Extraction Software (version 10.7.1.1; Agilent Technologies, Inc.) was used to process the original image and extract the data. The GeneSpring software (version 12.5; Agilent Technologies, Inc.) was used to analyze normalization and variance. Finally, all the differentially expressed lncRNAs were screened out with the selection criteria of fold change (FC) $>2$ and $\mathrm{P}<0.05$. 

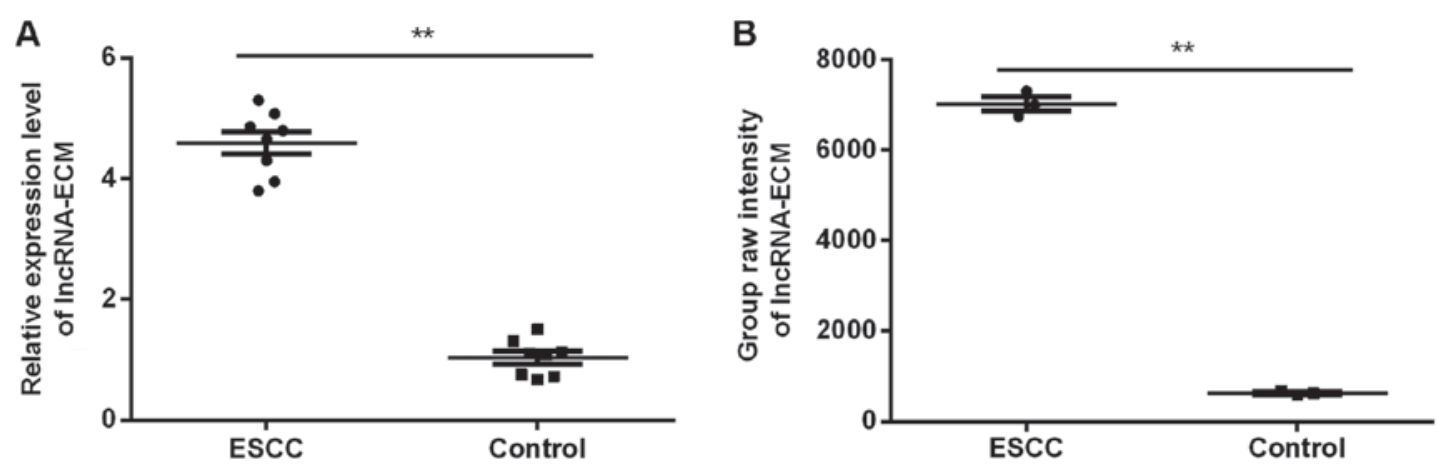

Figure 1. LncRNA-ECM analysis. (A) LncRNA-ECM were validated by reverse transcription-quantitative polymerase chain reaction in 8 patients with ESCC. (B) The chip original signal of lncRNA-ECM in 3 patients with ESCC. ${ }^{* *} \mathrm{P}<0.01$. ESCC, esophageal squamous cell carcinoma; Lnc, long-non coding; ECM, extracellular matrix.

Cell culture and transfection. The human ESCC cell lines TE-1, KYSE150, Eca109, KYSE30, EC9706 and the normal human esophageal mucosal epithelial HET-1A cell line were purchased from Shanghai Meixuan Biotechnology (Shanghai, China) and cultured in RPMI-1640 (Corning Inc., Corning, NY, USA) medium containing $10 \%$ fetal bovine serum (GE Healthcare Life Sciences, Logan, UT, USA) in $5 \% \mathrm{CO}_{2}$ at $37^{\circ} \mathrm{C}$. In the logarithmic growth phase, TE-1 and Eca109 cells were inoculated into 6-well plates $\left(4 \times 10^{5}\right.$ cells $\left./ \mathrm{ml}\right)$. When the cell healing degree reached 90-95\%, the plasmids of IncRNA-ECM silencing and overexpression were transfected into the cells by Lipofectamine 2000 transfection reagent (Invitrogen; Thermo Fisher Scientific, Inc.). The IncRNA-ECM overexpression plasmids were synthesized according to the NCBI reference sequence. PcDNA3.1 vectors were used as null controls. After transfection for $48 \mathrm{~h}$, the cells were used for the experiments. The sequences of si-lncRNA-ECM were as follows: si-IncRNA-ECM 1\#, sense, 5'-CAGGAAAGCCAUACCAUG ATT-3' and antisense, 5'-UCAUGGUAUGGCUUUCCU GTT-3'; si-lncRNA-ECM 2\# sense, 5'-CCUAUAGAGCGA CUGUCAATT-3' and antisense, 5'-UUGACAGUCGCUCUA UAGGTT-3'; si-lncRNA-ECM 3\# sense, 5'-CAGCAAACU CGUAGGUCAATT-3' and antisense, 5'-UUGACCUACGAG UUUGCUGTT-3' (Sangon Biotech, Shanghai, China).

Cell invasion and migration assays. Transwell plates (Costar, New York, NY, USA) were used for the Transwell assays. After $12 \mathrm{~h}$ of transfection, the cells were inoculated into the 24-well Transwell plate (Corning Inc.) at an inoculation volume of $4 \times 10^{3} /$ well. The membrane at the bottom of the upper chamber of the Transwell plate was coated with $100 \mu 1$ $(\sim 25 \mu \mathrm{g})$ diluted Matrigel (BD Biosciences, Franklin Lakes, $\mathrm{NJ}, \mathrm{USA}$ ) and dried at $4^{\circ} \mathrm{C} ; 50 \mu \mathrm{l}$ serum-free culture medium was added to each well and cultured at $37^{\circ} \mathrm{C}$ for $30 \mathrm{~min}$. To digest and collect transfected cells, the cells were suspended in serum-free medium at a density of $10^{5} / \mathrm{ml} ; 100 \mu$ l of the cell suspension was added to the upper chamber of the 24-well Transwell plate. Complete medium with $500 \mu 120 \%$ FBS was added to the lower chamber. The plates were incubated in an incubator with $5 \% \mathrm{CO}_{2}$ at $37^{\circ} \mathrm{C}$ for $24 \mathrm{~h}$, washed with PBS twice, the upper layer of the cells was wiped with a cotton swab, fixed for $20 \mathrm{~min}$ in methanol, dyed for $10 \mathrm{~min}$ with
$0.1 \%$ crystal violet solution, and rinsed with distilled water until there was no stain under the optical microscope. Finally, five random $\times 20$ visual fields were observed, and the number of tumor cells was counted. The migration assay was performed in a similar manner, without the addition of Matrigel. All the experiments were performed in triplicate.

Statistical analysis. Student's t-test was used to evaluate the significance of the difference between the two groups of lncRNA-ECM expression in ESCC at the tissue level and the cellular level. One-way ANOVA was used to compare the means of the multiple groups data. When the result is significant, the LSD post hoc test was then used to compare the means between two pairs in this group. The clinicopathological characteristics were analyzed by the Chi-squared test or Wilcoxon's rank-sum test. The correlations were assessed by Pearson's correlation analysis. Data are expressed as mean \pm standard deviation of three independent experiments. $\mathrm{P}<0.05$ was considered to indicate a statistically significant difference. All statistical analyses were performed by SPSS 22.0 (IBM Corp., Armonk, NY, USA).

\section{Results}

Microarray analysis. Among the 39 lncRNAs with significant differences (FC $>10)$ reported previously (12), due to the significant between-group difference, non-significant in-group difference (Fig. 1A) and higher abundance compared with its origin (Fig. 1B) in the chip, IncRNA-ECM was selected.

Potential functional analysis of IncRNA-ECM. Using Gene Ontology (GO) and Kyoto Encyclopedia of Genes and Genomes (KEGG) analysis, we found that IncRNA-ECM and its potential target genes are closely associated with cell cycle regulation, cell differentiation and other processes (Fig. 2A-C), including extracellular matrix receptor interaction, cell cycle regulation, adhesive junctions, and other multiple signaling pathways associated with cancer (Fig. 2D), and it may also be involved in the pathogenesis of ESCC.

Expression of IncRNA-ECM in ESCC tissues and association with clinicopathological parameters. To further investigate the biological behavior of IncRNA-ECM in ESCC, we examined 
A
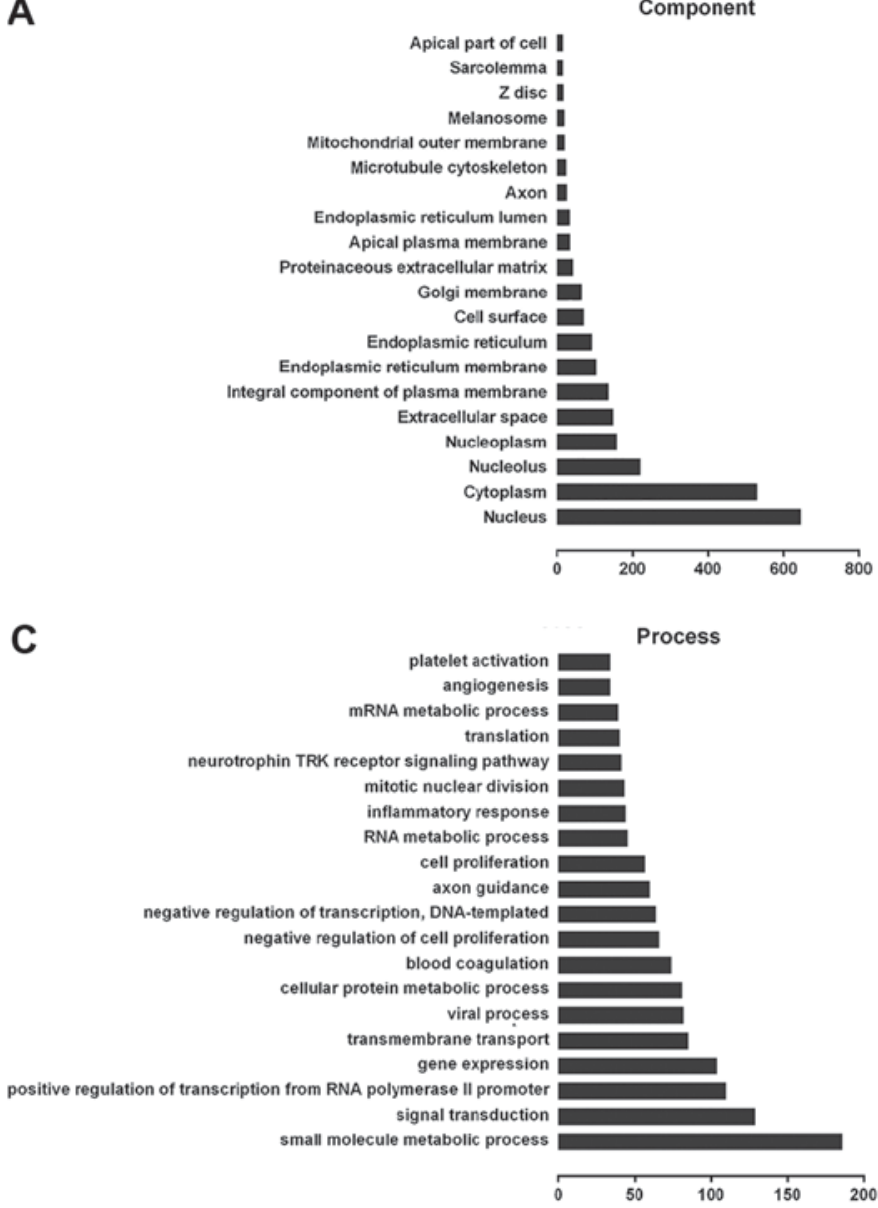

B

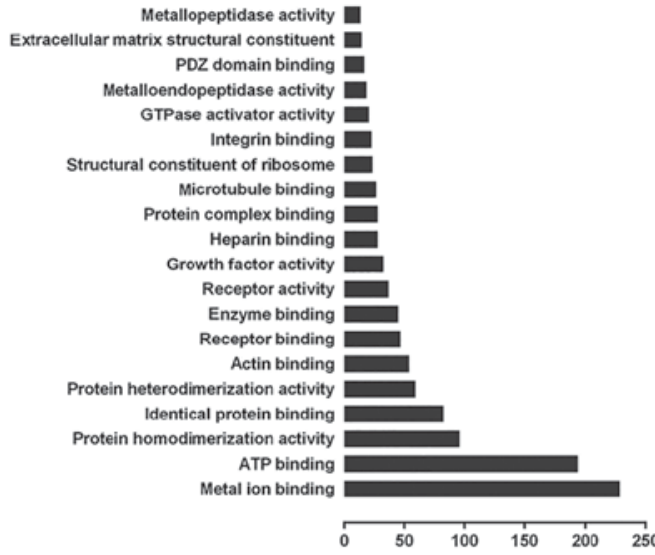

D

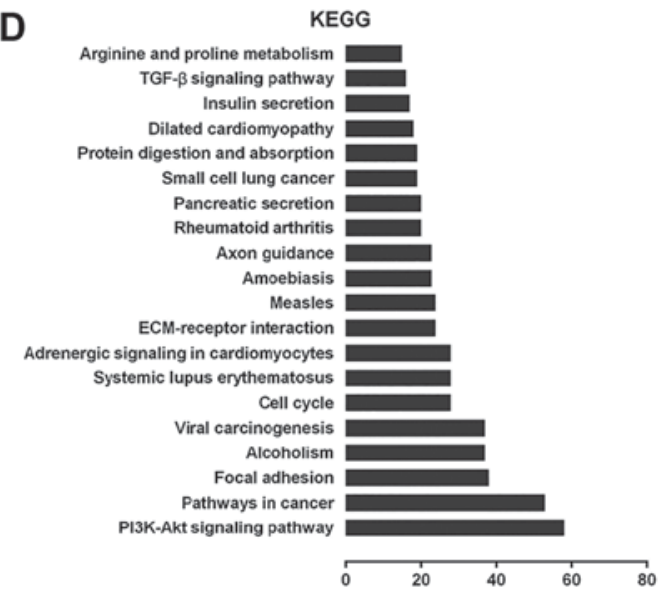

Figure 2. LncRNA-ECM GO and pathway analysis. (A) Cellular component of lncRNA-ECM GO terms. (B) Molecular function of lncRNA-ECM GO terms. (C) Biological process of lncRNA-ECM GO terms. (D) LncRNA-ECM pathway analysis. GO, Gene Ontology; Lnc, long-non coding; ECM, extracellular matrix.
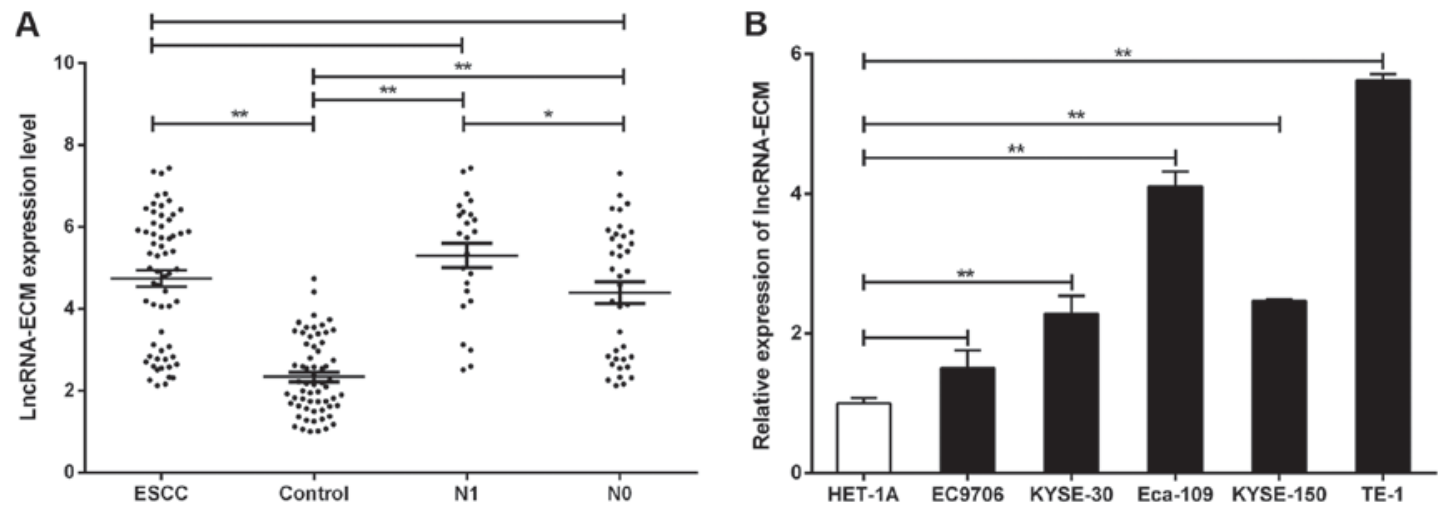

Figure 3. Expression of lncRNA-ECM in ESCC. (A) LncRNA-ECM was upregulated in ESCC tissues and patients with lymphatic metastasis (N1) had increased lncRNA-ECM expression. (B) Relative expression of lncRNA-ECM in a panel of ESCC cell lines. " $\mathrm{P}<0.05$ and ${ }^{* *} \mathrm{P}<0.01$. Lnc, long-non coding; ECM, extracellular matrix; ESCC, esophageal squamous cell carcinoma.

the expression of IncRNA-ECM in cancer tissues from $62 \mathrm{ESCC}$ patients and corresponding paracancerous tissues by RT-PCR. The results demonstrated that the expression of IncRNA-ECM in ESCC tissues was significantly higher compared with that in adjacent tissues (Fig. 3A), which was in agreement with the microarray results. The experiments on cultured ESCC cell lines also yielded similar results: Compared with the normal human esophageal mucosal epithelial HET-1A cell line, the expression of IncRNA-ECM was significantly higher in all human ESCC cell lines (TE-1, KYSE150, Eca109 and KYSE30; Fig. 3B). The clinicopathological characteristics of the 62 ESCC cases are listed in Table I. LncRNA-ECM was significantly associated with lymph node metastasis $(\mathrm{P}=0.029$; Table I and Fig. 3A) and TNM stage ( $\mathrm{P}=0.036$, Table I), but not with age, sex, tumor size, pathological differentiation or $\mathrm{T}$ stage (all $\mathrm{P}>0.05$; Table I). These findings indicate that 

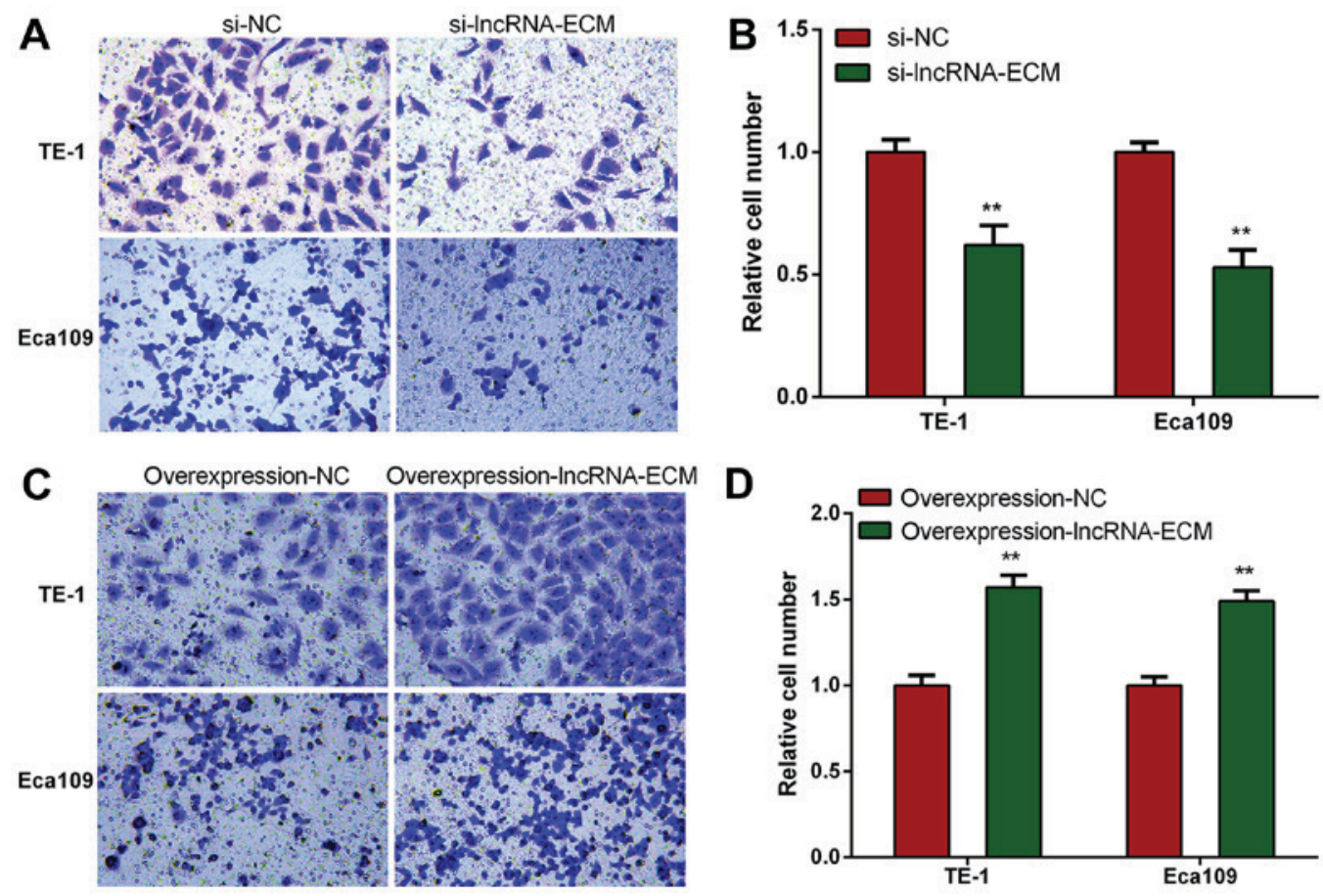

Figure 4. Knockdown of lncRNA-ECM reduces the invasion and migration of ESCC cells. (A and B) The cell invasion ability decreased following knock-down of lncRNA-ECM. (C and D) The cell migration ability decreased following knock-down lncRNA-ECM. ${ }^{* *} \mathrm{P}<0.01$. Magnification, $\mathrm{x} 200$. Lnc, long-non coding; ECM, extracellular matrix; NC, negative control; ESCC, esophageal squamous cell carcinoma.
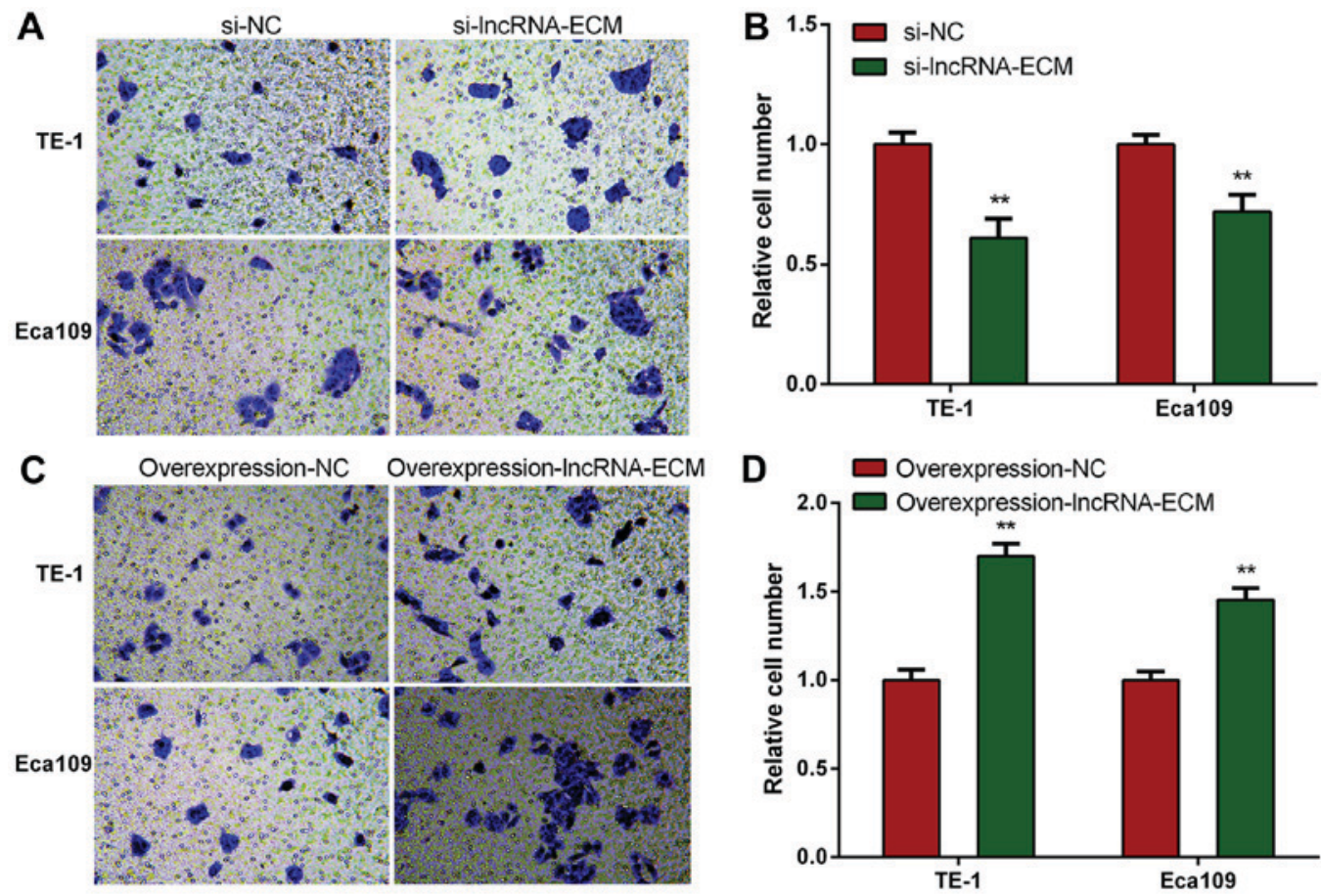

Figure 5. Overexpression of lncRNA-ECM increases the invasion and migration of ESCC cells. (A and B) The cell invasion ability increased following overexpression of lncRNA-ECM. (C and D) The cell migration ability increased following overexpression of lncRNA-ECM. *"P<0.01. Magnification, x200. Lnc, long-non coding; ECM, extracellular matrix; NC, negative control; ESCC, esophageal squamous cell carcinoma.

overexpression of IncRNA-ECM may be associated with ESCC metastasis.

Knockdown of IncRNA-ECM decreases invasion and migration of ESCC cells. To explore the function of IncRNA-ECM in ESCC invasion and migration, we selected two highest cell lines of TE-1 and Eca109 to perform loss or gain of function assays. We transfected the specific siRNA of IncRNA-ECM into TE-1 and Eca109 cell lines and utilized a classical Transwell assay to assess the effect of IncRNA-ECM on the invasion and migration. The results revealed that the invasion and migration of ESCC cell lines decreased significantly after lncRNA-ECM 

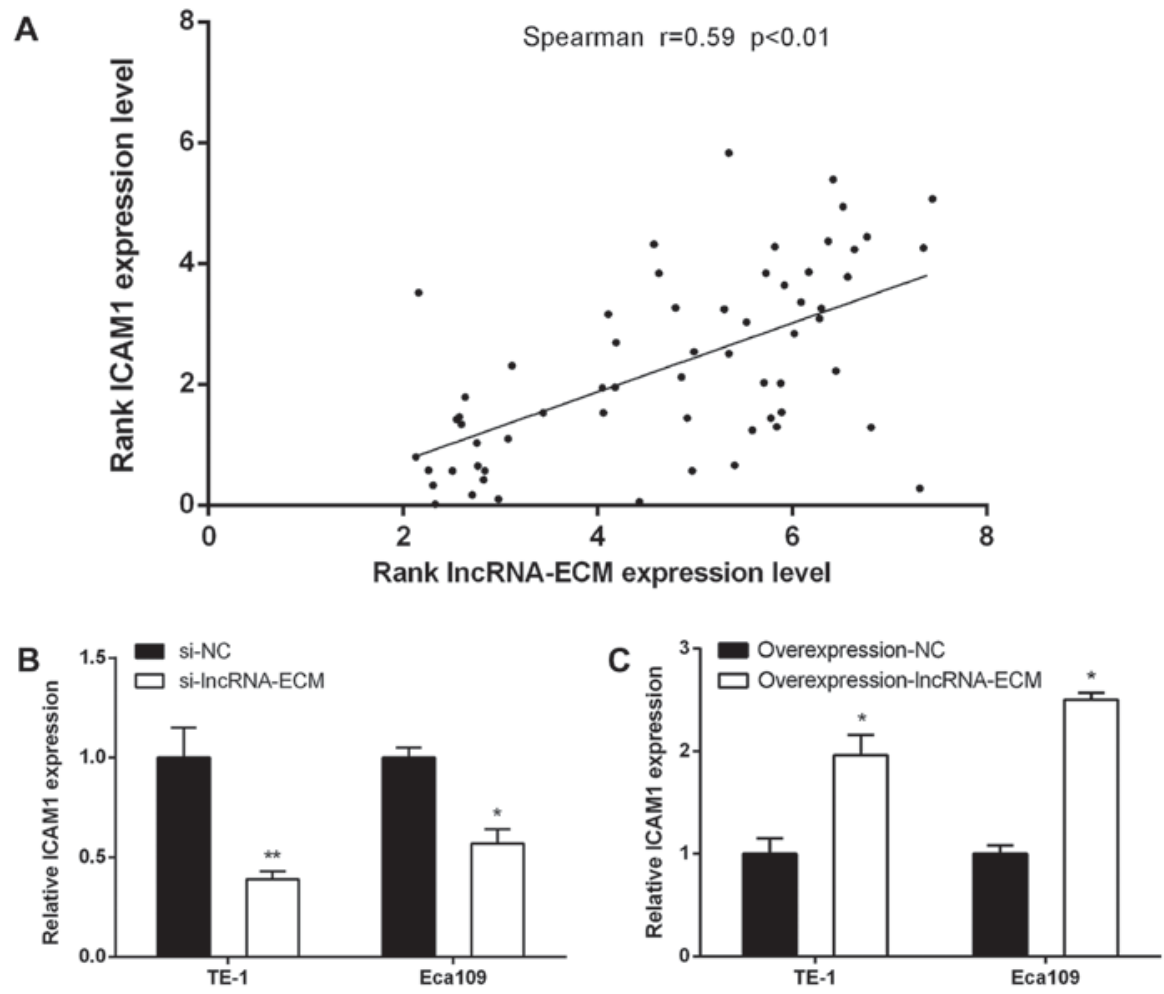

Figure 6. Correlation analysis between lncRNA-ECM and ICAM1. (A) Positive correlation between lncRNA-ECM and ICAM1 in ESCCs. (B) Following knock-down of lncRNA-ECM the level of ICAM1 decreases. (C) Following overexpression of lncRNA-ECM the level of ICAM1 increased. "P $<0.05$ and ${ }^{* *} \mathrm{P}<0.01$ vs. the NC group. Lnc, long-non coding; ECM, extracellular matrix; ICAM1, intercellular adhesion molecule 1; NC, negative control.

silencing (Fig. 4). The result indicated that lncRNA-ECM is involved in the regulation of ESCC cell invasion and migration.

Overexpression of IncRNA-ECM increases invasion and migration of ESCC cells. To elucidate whether IncRNA-ECM promotes ESCC cell invasion and migration, IncRNA-ECM overexpression plasmids were transfected into ESCC TE-1 and Eca109 cell lines, and the invasion and migration ability of the cell lines was evaluated after transfection. It was observed that the invasion and migration ability of ESCC cells was markedly increased in cells overexpressing lncRNA-ECM (Fig. 5). This result confirmed that IncRNA-ECM plays an important role in promoting ESCC cell migration.

LncRNA-ECM may regulate ICAMI in ESCC cells. As genes with similar spatial location are often the targets of lncRNA regulation (6), the location of IncRNA-ECM and nucleic acid sequences was analyzed by UCSC and BLAST, and we found that ICAM1 (intercellular cell adhesion molecule-1) is adjacent to lncRNA-ECM. We detected the expression level of ICAM1 in the 62 cases of ESCC using RT-qPCR in the present study, and the results demonstrated that the expression level of ICAM1 was positively correlated with lncRNA-ECM expression (Fig. 6A; Pearson's correlation, $\mathrm{R}=0.59, \mathrm{P}<0.01$ ). We further investigated whether the expression of ICAM1 changed according to the level of IncRNA-ECM in ESCC cells. It was observed that, after knocking down lncRNA-ECM, the expression of ICAM1 also decreased, whereas the level of ICAM1 increased following transfection of IncRNA-ECM overexpression plasmids (Fig. 6B and C), suggesting that ICAM1 may be a downstream target of lncRNA-ECM.

\section{Discussion}

Despite a slight decrease in its incidence in recent years, ESCC remains one of the most common malignant tumors in China. Due to its high invasiveness and propensity for metastasis, the prognosis of ESCC is not satisfactory. Over the last decades, the association between biomolecules and cancer has attracted extensive attention, and a number of studies on ESCC have been performed $(13,14)$. However, only few sensitive and specific biomarkers for ESCC have been identified. Therefore, active research on the development of effective molecular markers will be beneficial in improving the diagnosis and prognosis of esophageal cancer.

With the rapid development of genome microarray and genome sequencing technology, a type of transcription without protein encoding, originally considered to be non-RNA genome encoding transcription 'noise', has become a research focus in recent years (15). Considering the abundance, variability, function and mechanism of lncRNAs, they may be the regulatory core of RNA, and an increasing number of studies have demonstrated that the differential expression of IncRNAs is closely associated with tumorigenesis and tumor development $(7,8,16)$, which provides a new basis for understanding the mechanism underlying these processes. Since lncRNA AFAP1-AS1 was found to be highly expressed in esophageal adenocarcinoma and silencing its expression significantly inhibited the proliferation, invasion and metastasis of esophageal adenocarcinoma cell lines (17), the research of IncRNAs and ESCC has attracted the attention of many scholars, and a few types of IncRNAs have been identified in ESCC. For example, IncRNA AFAP1-AS1 (9), 
HOTAIR (10) and POU3F3 (18) are highly expressed in ESCC, and their high expression can promote the development of ESCC and adversely affect the prognosis. Following knockdown of these lncRNAs, the proliferation, invasion and migration ability of ESCC cells was significantly inhibited. In addition, the expression of IncRNA-LET (19), UCA1 (20) and LOC285194 (21) is low in ESCC tissues, and their expression level is inversely associated with tumor size, TNM stage, lymph node metastasis, distant metastasis, survival rate and prognosis, whereas their overexpression can inhibit the migration of ESCC cells.

LncRNA-ECM (lncRNA ENST00000589379) is a newly discovered lncRNA, which exhibited high expression in the ESCC gene chip (12). Sequence analysis demonstrated that lncRNA-ECM was composed of 3,218 bp, and there were a number of cis-regulatory elements, binding sites and hypersensitive sites of DNase I in the region of the chromosome, indicating that the transcription status of this region was relatively active. There are abundant DNA hypermethylation regions and epigenetic regulatory loci in this region, such as histone H3K4me1, H3K4me3 and H3K36me3, among others. To some extent, histone modification and DNA methylation play important roles in the development and progression of ESCC $(22,23)$, suggesting the functional significance of IncRNA-ECM. In the present study, we investigated the expression of IncRNA-ECM in ESCC at the cellular and tissue level. Combining the lncRNA microarray data and RT-PCR results, the expression of IncRNA-ECM in ESCC tissues was found to be significantly higher compared with that in the corresponding paracancerous tissues and nomal control cells, indicating that IncRNA-ECM may be a biomarker for ESCC detection. In addition, we found that the expression level of lncRNA-ECM in cancers with lymph node metastasis was significantly higher. The results demonstrated that the expression of IncRNA-ECM was correlated with lymph node metastasis. We also investigated the association between the expression of IncRNA-ECM and TNM stage, and observed that a higher expression of lncRNA-ECM in patients with ESCC was associated with a more advanced TNM stage. Therefore, these findings suggest that lncRNA-ECM may be a potential marker for ESCC prognosis. Subsequently, we transfected siRNA plamids with lncRNA-ECM knockdown into ESCC TE-1 and Eca109 cells and found that the invasion and migration ability of the two cell lines was diminished; by contrast, after transfecting lncRNA-ECM overexpression plasmids, the invasion and migration ability of ESCC cells was enhanced, suggesting lncRNA-ECM plays an important role in ESCC metastasis and may be a characteristic molecule for diagnosing ESCC and predicting its prognosis.

LncRNA signaling is associated with integrin pathways, extracellular pathways and local adhesion pathways. ICAM1, a member of the immunoglobulin superfamily (IGSF), is an important adhesion molecule (24) involved in cell metastasis, differentiation and proliferation. Recently, ICAM1 was reported to serve as a liver cancer and ESCC stem cell marker $(25,26)$; it can also promote ESCC epithelial-to-mesenchymal transition (EMT) (27) and cause metastasis of ESCC cells by regulating the expression of tumor metastasis-related genes, such as P53 (26). We predicted the possible role of ICAM1 as a target gene for lncRNA-ECM according to bioinformatics analysis.
The expression of ICAM1 in ESCC tissues was detected by RT-PCR, and its expression level was found to be positively correlated with the expression of IncRNA-ECM. Furthermore, after knocking down lncRNA-ECM in ESCC cells, the level of ICAM1 decreased, while the expression level of ICAM1 increased following lncRNA-ECM overexpression. These data indicate that IncRNA-ECM plays a regulatory role in promoting lymph node metastasis in ESCC, and this regulation may be mediated by ICAM1.

In conclusion, we investigated the biological behavior of lncRNA-ECM in ESCC and found it to be overexpressed in ESCC tissues. It may be deduced that IncRNA-ECM plays an important role in oncogenesis and progression of ESCC by regulating ICAM1, and it may promote ESCC metastasis. Therefore, lncRNA-ECM may be a new biomarker for diagnosing ESCC and predicting patient prognosis, and it may also represent a novel molecular target for the treatment of ESCC.

\section{Acknowledgements}

Not applicable.

\section{Funding}

The present study was supported by the Jiangsu Provincial Medical Innovation Team (grant no. CXTDA2017042) of Jiangsu Province, and the 333 Plan Foundation (grant no. BRA2017173) of Jiangsu Province, China.

\section{Availability of data and materials}

The datasets used and/or analyzed during the current study are available from the corresponding author on reasonable request.

\section{Authors' contributions}

JY was responsible for writing the manuscript and performing the lncRNA-ECM cell experiments. XS was responsible for collecting the ESCC and matched para-cancerous tissues and performing the RT-qPCR experiments. HL, JX and SS were responsible for collecting the ESCC and matched para-cancerous tissues. JXH wrote the paper and performed the bioinformatics analysis. ML assisted with the experiments and revised the paper.

\section{Ethics approval and consent to participate}

The study was approved by the Ethics Committee of Taizhou People's Hospital affiliated to Nantong University and written informed consent was obtained from all participants.

\section{Patient consent for publication}

Not applicable.

\section{Competing interests}

\section{References}

1. Chen W, Zheng R, Zeng H, Zhang S and He J: Annual report on status of cancer in China, 2011. Chin J Cancer Res 27: 2-12, 2015. 
2. Jemal A, Bray F, Center MM, Ferlay J, Ward E and Forman D Global cancer statistics. CA Cancer J Clin 61: 69-90, 2011.

3. Bogoevski D, Onken F, Koenig A, Kaifi JT, Schurr P, Sauter G, Izbicki JR and Yekebas EF: Is it time for a new TNM classification in esophageal carcinoma? Ann Surg 247: 633-641, 2008.

4. Kayani B,Zacharakis E, Ahmed K and Hanna GB: Lymph node metastases and prognosis in esophageal carcinoma-a systematic review. Eur J Surg Oncol 37: 747-753, 2011.

5. Mercer TR, Dinger ME and Mattick JS: Long non-coding RNAs: Insights into functions. Nat Rev Genet 10: 155-159, 2009.

6. Engreitz JM, Pandya-Jones A, McDonel P, Shishkin A, Sirokman K, Surka C, Kadri S, Xing J, Goren A, Lander ES, et al: The Xist lncRNA exploits three-dimensional genome architecture to spread across the X chromosome. Science 341: 1237973 , 2013.

7. Liu B, Sun L, Liu Q, Gong C, Yao Y, Lv X, Lin L, Yao H, Su F, $\mathrm{Li} \mathrm{D}$, et al: A cytoplasmic NF- $\mathrm{BB}$ interacting long noncoding RNA blocks I $\kappa \mathrm{B}$ phosphorylation and suppresses breast cancer metastasis. Cancer Cell 27: 370-381, 2015.

8. Xing Z, Lin A, Li C, Liang K, Wang S, Liu Y, Park PK, Qin L, Wei Y, Hawke DH, et al: lncRNA directs cooperative epigenetic regulation downstream of chemokine signals. Cell 159: $1110-1125,2014$

9. Zhou XL, Wang WW, Zhu WG, Yu CH, Tao GZ, Wu QQ Song YQ, Pan P and Tong YS: High expression of long non-coding RNA AFAP1-AS1 predicts chemoradioresistance and poor prognosis in patients with esophageal squamous cell carcinoma treated with definitive chemoradiotherapy. Mol Carcinog 55: 2095-2105, 2016.

10. Chen FJ, Sun M, Li SQ, Wu QQ, Ji L, Liu ZL, Zhou GZ, Cao G, Jin L, Xie HW, et al: Upregulation of the long non-coding RNA HOTAIR promotes esophageal squamous cell carcinoma metastasis and poor prognosis. Mol Carcinog 52: 908-915, 2013.

11. Song W and Zou SB: Prognostic role of lncRNA HOTAIR in esophageal squamous cell carcinoma. Clin Chim Acta 463: 169-173, 2016

12. Yao J, Huang JX, Lin M, Wu ZD, Yu H, Wang PC, Ye J, Chen P, $\mathrm{Wu} \mathrm{J}$ and Zhao GJ: Microarray expression profile analysis of aberrant long noncoding RNAs in esophageal squamous cell carcinoma. Int J Oncol 48: 2543-2557, 2016.

13. Liu M, Hu Y, Zhang MF, Luo KJ, Xie XY, Wen J, Fu JH and Yang H: MMP1 promotes tumor growth and metastasis in esophageal squamous cell carcinoma. Cancer Lett 377: 97-104, 2016.

14. Liu L, Lin C, Liang W, Wu S, Liu A, Wu J, Zhang X, Ren P, Li $\mathrm{M}$ and Song L: TBL1XR1 promotes lymphangiogenesis and lymphatic metastasis in esophageal squamous cell carcinoma. Gut 64: 26-36, 2015.

15. Derrien T, Johnson R, Bussotti G, Tanzer A, Djebali S, Tilgner H, Guernec G, Martin D, Merkel A, Knowles DG, et al: The GENCODE v7 catalog of human long noncoding RNAs: Analysis of their gene structure, evolution, and expression. Genome Res 22: 1775-1789, 2012.
16. Svoboda M, Slyskova J, Schneiderova M, Makovicky P, Bielik L, Levy M, Lipska L, Hemmelova B, Kala Z, Protivankova M, et al: HOTAIR long non-coding RNA is a negative prognostic factor not only in primary tumors, but also in the blood of colorectal cancer patients. Carcinogenesis 35: 1510-1515, 2014.

17. Wu W, Bhagat TD, Yang X, Song JH, Cheng Y, Agarwal R, Abraham JM, Ibrahim S, Bartenstein M, Hussain Z, et al: Hypomethylation of noncoding DNA regions and overexpression of the long noncoding RNA, AFAP1-AS1, in Barrett's esophagus and esophageal adenocarcinoma. Gastroenterology 144 956-966.e4, 2013.

18. Li W, Zheng J, Deng J, You Y, Wu H, Li N, Lu J and Zhou Y: Increased levels of the long intergenic non-protein coding RNA POU3F3 promote DNA methylation in esophageal squamous cell carcinoma cells. Gastroenterology 146: 1714-1726.e5, 2014.

19. Wang PL, Liu B, Xia Y, Pan CF, Ma T and Chen YJ: Long non-coding RNA-Low Expression in Tumor inhibits the invasion and metastasis of esophageal squamous cell carcinoma by regulating p53 expression. Mol Med Rep 13: 3074-3082, 2016.

20. Wang X, Gao Z, Liao J, Shang M, Li X, Yin L, Pu Y and Liu R lncRNA UCA1 inhibits esophageal squamous-cell carcinoma growth by regulating the Wnt signaling pathway. J Toxicol Environ Health A 79: 407-418, 2016.

21. Tong YS, Zhou XL, Wang XW, Wu QQ, Yang TX, Lv J, Yang JS, Zhu B and Cao XF: Association of decreased expression of long non-coding RNA LOC285194 with chemoradiotherapy resistance and poor prognosis in esophageal squamous cell carcinoma. J Transl Med 12: 233, 2014

22. Hu C, Liu M, Zhang W, Xu Q, Ma K, Chen L, Wang Z, He S, Zhu $\mathrm{H}$ and $\mathrm{Xu}$ N: Upregulation of KLF4 by methylseleninic acid in human esophageal squamous cell carcinoma cells: Modification of histone $\mathrm{H} 3$ acetylation through HAT/HDAC interplay. Mol Carcinog 54: 1051-1059, 2015.

23. Kishino T, Niwa T, Yamashita S, Takahashi T, Nakazato H, Nakajima T, Igaki H, Tachimori Y, Suzuki Y and Ushijima T: Integrated analysis of DNA methylation and mutations in esophageal squamous cell carcinoma. Mol Carcinog 55: 2077-2088, 2016

24. Kluger HM, Hoyt K, Bacchiocchi A, Mayer T, Kirsch J, Kluger Y, Sznol M, Ariyan S, Molinaro A and Halaban R: Plasma markers for identifying patients with metastatic melanoma. Clin Cancer Res 17: 2417-2425, 2011.

25. Liu S, Li N, Yu X, Xiao X, Cheng K, Hu J, Wang J, Zhang D, Cheng S and Liu S: Expression of intercellular adhesion molecule 1 by hepatocellular carcinoma stem cells and circulating tumor cells. Gastroenterology 144: 1031-1041.e10, 2013.

26. Tsai ST, Wang PJ, Liou NJ, Lin PS, Chen CH and Chang WC: ICAM1 is a potential cancer stem cell marker of esophageal squamous cell carcinoma. PLoS One 10: e0142834, 2015.

27. Mani SA, Guo W, Liao MJ, Eaton EN, Ayyanan A, Zhou AY, Brooks M, Reinhard F, Zhang CC, Shipitsin M, et al: The epithelial-mesenchymal transition generates cells with properties of stem cells. Cell 133: 704-715, 2008. 